\title{
SIESTA: a High Current Ion Source for Erosion and Retention Studies
}

\author{
R. Arredondo ${ }^{\text {a),b) }{ }_{,}, \text {M. Oberkofler }}{ }^{\text {a) }}$, K. Schmid ${ }^{\text {a) }}$, T. Schwarz-Selinger ${ }^{\text {a) }}$, W. Jacob ${ }^{\text {a) }}$, R. Neu ${ }^{\text {a),b) }}$ \\ a) Max-Planck-Institut für Plasmaphysik, Boltzmannstrasse 2, Garching D-85748, Germany \\ b) Technische Universität München, Boltzmannstrasse 15, Garching D-85748, Germany \\ *rodrigo.arredondo@ipp.mpg.de
}

\begin{abstract}
SIESTA (Second Ion Experiment for Sputtering and TDS Analysis) is a high-current ion source for erosion and retention studies with focus on wall materials for fusion devices. The system is composed of a DuoPIGatron type ion source, three consecutive grids for ion extraction as well as acceleration and beam focusing, a differential pumping stage, a dipole magnet for mass filtering, a quadrupole doublet lens, a target chamber, a load-lock and a chamber for thermal desorption spectrometry. The acceleration potential of the source can be varied between $500 \mathrm{~V}$ and $10 \mathrm{kV}$. The target chamber has a base pressure of $10^{-8}$ mbar, and an operating pressure of $5 \times 10^{-7} \mathrm{mbar}$. The target can be rotated to study angle-dependent effects and can be heated via electron-impact heating up to $1300 \mathrm{~K}$ for high temperature erosion and implantation studies. The target chamber is equipped with an in-situ with a magnetic suspension balance. The operating parameters of the ion source were mapped to achieve the maximum ion current at the target for various gas species and accelerating potentials. The beam emittance for a $\mathrm{D}_{3}{ }^{+}$ion beam was measured after deflection in the dipole magnet. This was used for ion beam simulations, which were instrumental for the design of the quadrupole lenses. If the quadrupole doublet is used, the ion flux to the target is increased by up to a factor of 4 . Additionally, the relative population of neutral particles present in the beam at the target was quantified, and is equal to $0.8 \%$ when averaged over the measurement positions. The typical beam footprint at the target under normal incidence has an area of $0.5 \mathrm{~cm}^{2}$. The ion current reaching the target increases with the accelerating potential. Due to this effect, the ion flux density at the target in the low-ion-impact-energy range can be increased by operating the source at a higher extraction potential and by applying a (decelerating) potential to the target. Ion impact energies as low as $200 \mathrm{eV} / \mathrm{D}$ are achieved this way with a $\mathrm{D}_{3}{ }^{+}$ current of $100 \mu \mathrm{A}$ when focusing the beam with the quadrupole doublet lens, equating to a D particle flux density of $3.7 \times 10^{19} \mathrm{~m}^{-}$ ${ }^{2} \mathrm{~s}^{-1}$. At ion impact energies of $2 \mathrm{keV} / \mathrm{D}$, the maximum achievable flux density with $\mathrm{D}_{3}^{+}$is $6 \times 10^{19} \mathrm{D} \mathrm{m}^{-2} \mathrm{~s}^{-1}$. Experimental determination of sputter yields was performed via ex-situ weight loss measurement for bulk Au samples, showing reasonably good agreement with simulations and experimental data from literature.
\end{abstract}

Published in Review of Scientific Instruments: Received $7^{\text {th }}$ of May 2018

Accepted for publication $8^{\text {th }}$ of September 2018

Published $1^{\text {st }}$ of October 2018

Rev.Sci. Instrum. 89:10 (2018). https://doi.org/10.1063/1.5039156

\section{Introduction}

The choice of plasma-facing material is highly significant for future fusion devices, as it imposes a series of safety, operational and economic constraints on the device. In particular, three issues have been identified as critical: the lifetime of plasma-facing components (PFCs), dust production from the erosion of PFCs and tritium retention in the inner walls of the device [1].

In the case of the first wall, erosion is caused predominantly by an intense flux of neutral particles, which are neutralized close to the plasma edge and are, therefore, no longer confined by the magnetic field. This neutral particle flux density is estimated to be in the range of $10^{19}-10^{21} \mathrm{~m}^{-2} \mathrm{~s}^{-1}$, with impinging particle energies normally in the $8-300 \mathrm{eV}$ range [1]. In this energy range, erosion is driven by physical sputtering [2] and can be accurately simulated by Coulomb interactions [3]. This process can also be studied experimentally, either in plasma devices which emulate the conditions of the plasma edge of a fusion reactor, or with the use of an ion source. Plasma devices allow for high particle flux densities to the target surface in the desired particle energy range. However, the presence of impurities, multiple ion species and a not easily determinable flux of energetic neutrals along with the intrinsic energy spread of the particles can make the quantitative analysis of results difficult. In contrast, ion sources have a number of distinct advantages over plasma devices, producing a mono-energetic beam which can be mass-filtered with magnetic sector fields, allowing for well-defined experiments. Historically, these machines have achieved significantly lower particle flux densities 
than their plasma counterparts, leading to longer exposure times to reach the desired fluence.

SIESTA (Second Ion Experiment for Sputtering and TDS Analysis) helps to bridge this gap, aiming to provide higher particle flux densities without losing the advantages of the ion source setup. SIESTA is the successor of the pre-existing HSQ (in German, HochStromQuelle) [4], which during its over 40 years of operation at the Max Planck Institute of Plasma Physics in Garching was instrumental in over 200 publications. At SIESTA, a DuoPIGatron type ion source is employed to provide particle flux densities of several $10^{19} \mathrm{~m}^{-2} \mathrm{~s}^{-1}$ to the target with a mono-energetic, mass-filtered ion beam (typically $\mathrm{D}_{3}^{+}$), with a final operating energy range of $200 \mathrm{eV}-10 \mathrm{keV}$. The device is described in section 2 , and section 3 details the results of the characterization of SIESTA. The sputter yield of Au by deuterium atoms with an impact energy of $2 \mathrm{keV}$ was measured and contrasted with simulations and other experimental data, shown in section 4. Finally, the main conclusions are summarized in section 5 .

\section{Description of the experimental setup}

A principle overview of the individual components of SIESTA is presented in this section. The device is described in more detail in the supplementary material. Fig. 1 shows a bird's eye view of SIESTA along with its main elements.

A plasma is generated inside the DuoPIGatron-type ion source [5] by way of electron ionization of a gas with a heated filament. The ion source is set to a high (positive) potential of maximum $10 \mathrm{kV}$, which determines the extracted ion energy. A series of three grids, the first at ion source potential and the last at ground potential, separates the ion source from the rest of the beam path. The ions that diffuse towards the grids are accelerated by the potential difference in the space between them, leading to a stationary ion beam of the desired energy. The intermediate grid is negatively biased and serves to repel electrons away from the positively-biased ion source.

Due to the large ion current density at the exit of the ion source, the space-charge of the beam would lead to strong beam divergence. However, low-energy (relative to the extracted ion energy) electrons produced in charge-exchange reactions downstream from the source are trapped in the potential well of the ion beam, negating the effect of space-charge. This is known as space-charge compensation [6].

The neutral gas from the ion source is mostly pumped in the differential pumping stage. The beam diameter is limited at the exit of the differential pumping stage by a $\varnothing 16 \mathrm{~mm}$ Mo aperture. A pneumatically-actuated beam-stopper is located directly behind this aperture. The beam-stopper is equipped with a Faraday cup to measure the impinging ion current at this location.

Behind the Mo aperture and with the beam stopper retracted, the beam passes through the dipole magnet. Each ion species present in the beam is deflected, with a radius of curvature proportional to the square root of its mass, thereby splitting the beam. The magnetic field is varied to allow only the desired ion species to exit through the opening to the target chamber, situated at a $44^{\circ}$ angle from the dipole magnet entrance. A maximum beam rigidity of $0.091 \mathrm{Tm}$ can be handled by the dipole magnet, corresponding to a $10 \mathrm{keV} \mathrm{Ar}^{+}$beam deflected to the target with a radius of curvature of $150 \mathrm{~mm}$. The maximum induction of the magnet is $0.7 \mathrm{~T}$. Another experiment, PERMEX (Permeation Experiment), is planned for the left exit of the dipole magnet. 


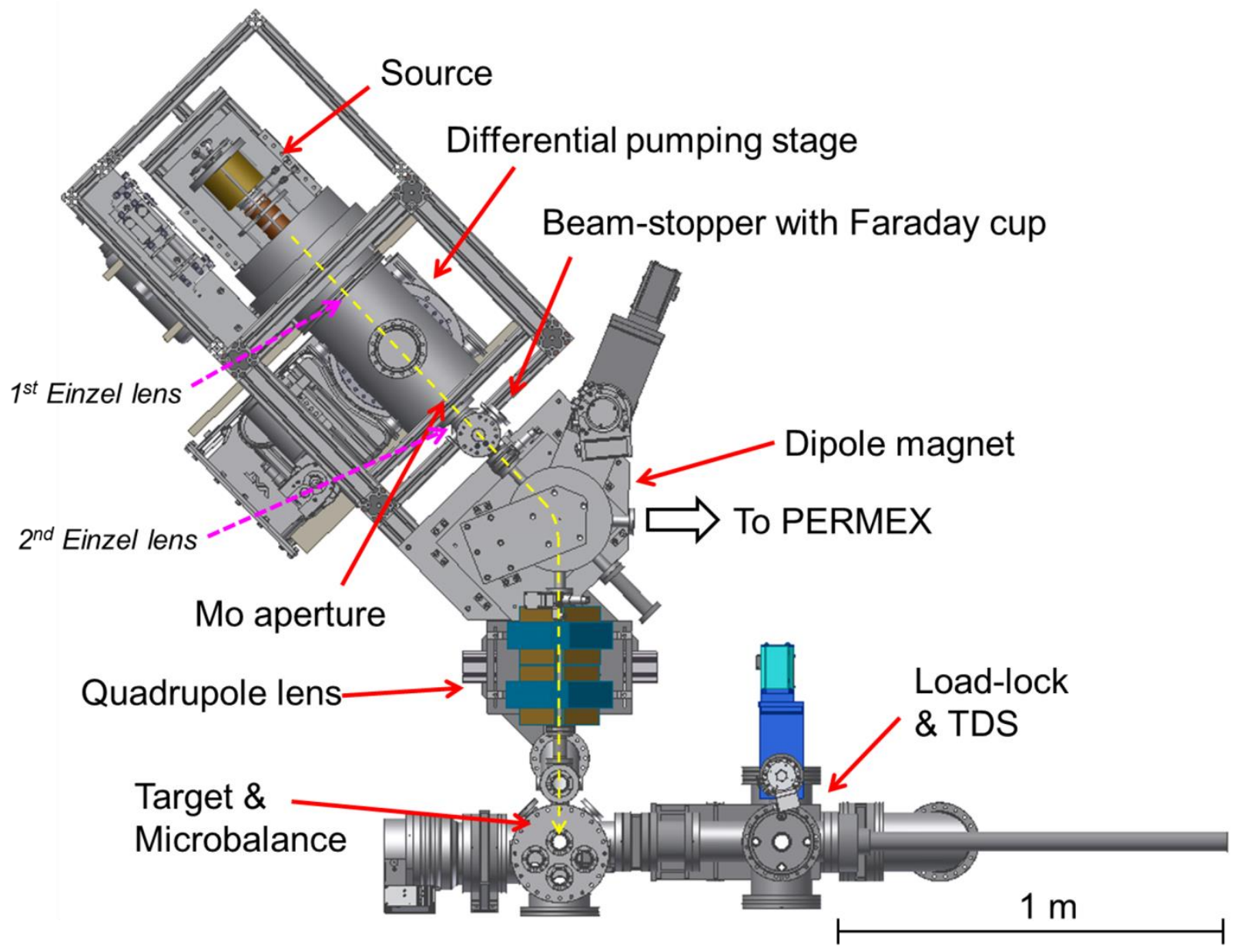

Figure 1: CAD bird's-eye view of SIESTA. The yellow dashed arrow indicates the ion trajectory from the ion source to the target. The einzel lenses (marked with dashed magenta arrows) were later removed. The new version of the ion-driven permeation experiment PERMEX will be located at the exit of the dipole magnet labeled "To PERMEX". Color designations refer to the online version only.

The target is housed in a double cage that serves as an electrical screening element and as a secondary electron catcher for the ion current measurement at the target. On its trajectory, the beam passes through an antechamber and is cropped by an adjustable aperture into a circular or elongated shape. Finally, it passes through openings in the double cage before impinging on the target.

The target can be rotated and heated via electronimpact heating up to $1300 \mathrm{~K}$. It can be set to a positive potential in order to decelerate incoming ions and thus study erosion at low (i.e., sub-keV) particle energies while continuing to operate the ion source at a high accelerating potential, which produces high particle flux densities. The sample temperature is measured with a Mauer-type TMRS $95 / 105^{1}$ infrared pyrometer and a type-K thermocouple connected to the target. A novel clamping mechanism was developed to ensure adequate contact between target and thermocouple, and is described in more detail in the supplementary material. A newly-installed magnetic suspension balance is envisaged for in-situ weight loss measurement of the sample. However, the balance has not yet been sufficiently characterized for general use. This will be the focus of future work to expand the capabilities of SIESTA. The target and double cage are shown in figure 2 .

\footnotetext{
${ }^{1}$ Trademark of Dr. Georg Maurer GmbH Optoelektronik.
} 
Samples can be loaded via a lateral, separatelypumped load-lock with a horizontal manipulator. A vertical manipulator at the load-lock can be used to transfer the sample to the Thermal Desorption Spectroscopy (TDS) chamber, located directly underneath. This enables in-vacuo TDS measurements of, e.g., released hydrogen isotopes directly after implantation

All vacuum chambers are pumped differentially by dedicated turbomolecular pumps. Base pressures in the range of $10^{-8}$ mbar are achieved in all vacuum chambers. The TDS chamber was baked to achieve a base pressure below $5 \times 10^{-9}$ mbar. Pumping at the dipole magnet has been improved with respect to the HSQ [4] with the addition of a dedicated turbomolecular pump. Due to this and other changes to the vacuum system, the pressure in the target chamber during ion bombardment was improved respective to the HSQ to $5 \times 10^{-7}$ mbar versus 7 $10 \times 10^{-7}$ mbar [4].

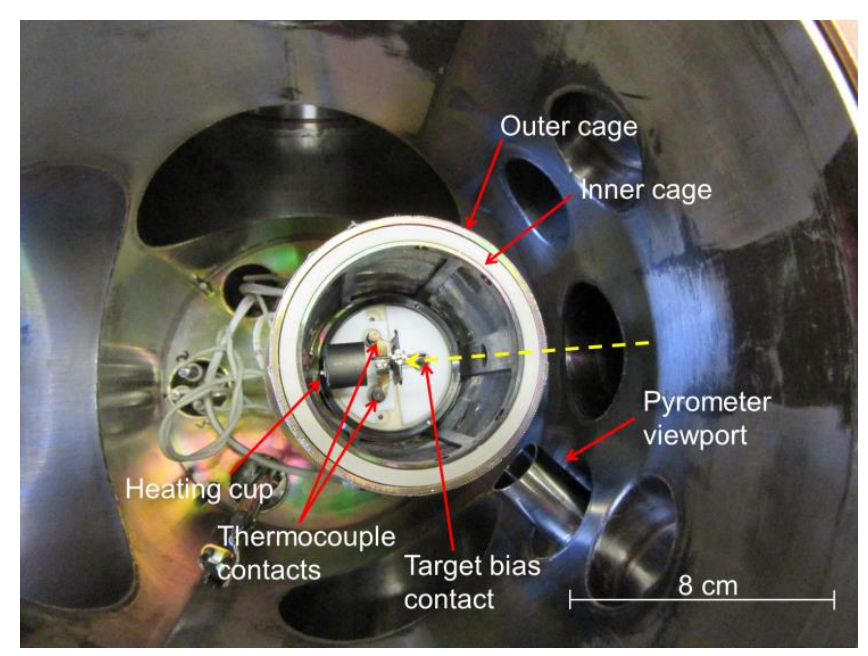

Figure 2: Top view of target chamber interior showing the double cage, pyrometer viewport, heating cup and target holder with thermocouple and target bias contacts. The beam defining aperture is absent. The beam direction is from right to left as indicated by the yellow dashed arrow. (Color online).

\section{Characterization}

\subsection{Mapping of source parameters}

The ion source was fully characterized for use with deuterium. Hydrogen (protium), hydrogen-helium and hydrogen-argon plasmas were also successfully produced. The latter two behave similarly to hydrogen and deuterium, unless stated otherwise. More details on the characterization of SIESTA are available in the supplementary material.

With the goal of maximizing the ion current reaching the target, parameter scans were performed to study the effect on the ion current of the extraction voltage, the pressure in the differential pumping stage (controlled by varying the neutral gas throughput) and the ion source operating parameters (source magnet, filament and arc currents). Additionally, the optimal pressure in the differential pumping stage and the optimal intermediate grid voltage were also systematically studied as a function of the extraction voltage. For these tests, the ion current was measured with the Faraday cup located at the beam-stopper, between the differential pumping stage and the dipole magnet (fig. 1). A vertical cross section of the beam profile was measured with an aperture of $\varnothing 1 \mathrm{~mm}$ as explained further below.

The ion source operating parameters used in the HSQ were taken as reference values, as they were proven to enable reliable, steady state operation while fulfilling the experimental requirements of the device. Said reference values are listed in the supplementary material.

Fig. 3 shows the maximum ion current measured with the Faraday cup at the beam-stopper with the extraction voltage ranging from 0.7 to $15 \mathrm{kV}$ when optimizing the gas flow to the ion source. The data were fitted by applying $I \propto U^{3 / 2}$, i.e., proportional to Child-Langmuir law [7], showing good agreement. The proportionality factor is the perveance measured at the beam-stopper [8]. It must be noted that the Child-Langmuir law only describes the space-chargelimited current that is extracted from the source, while the data points correspond to the current measured at the beam stopper, i.e., after the beam has passed through the differential pumping stage and has been partly cut off by the Mo aperture. Under these conditions, the variation of the ion source operating 
parameters shows no effect on the extracted ion current within the experimental uncertainty. However, the source is not space-charge limited, since the perveance of the source is significantly smaller than the maximum perveance given by the Child-Langmuir law and the ion current emitted from the source varies with the gas throughput [8].

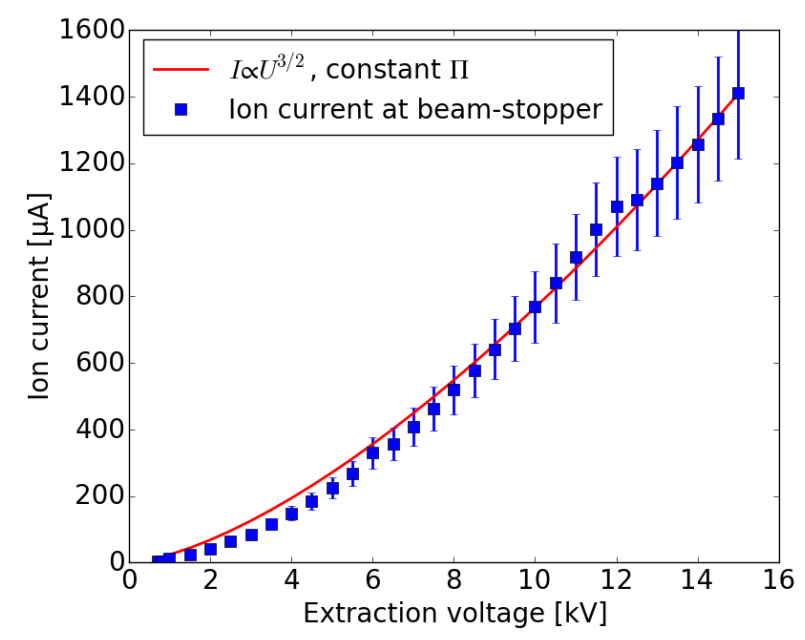

Figure 3: Ion current measured at the beam-stopper with increasing extraction voltage, fitted with a curve of constant perveance [8]. The increase in the size of the error bars for ion currents above 1 $\mathrm{mA}$ is due to a change in the measurement range of the current integrator. (Color online).

The correlation of the optimal intermediate grid voltage for maximum ion current at the target with the extraction voltage was also investigated. Since the negatively-biased intermediate grid screens the ion source from back-streaming electrons, the optimal grid voltage is proportional to the extraction potential. The proportionality constant is approximately 0.01 . At less negative biases, the intermediate grid does not sufficiently screen the ion source from back-streaming electrons. At more negative biases, the intermediate grid de-focuses the ion beam, leading to lower measured ion currents.

The ion current variation around the optimal pressure in the differential pumping stage was also studied. At pressures below the optimum, higher ion currents are emitted from the ion source, but due to stronger beam divergence, the current reaching the target is lower. At pressures above the optimum, the current emitted from the source is lower and a larger fraction of the beam is neutralized, predominantly in the first differential pumping stage, leading to lower ion currents at the target. The pressure optimum was typically around $10^{-4}$ mbar.

Dependences between the intermediate grid voltage and the pressure optimum and other source parameters (current through ion source magnet, filament and arc current) were also tested, but none were found.

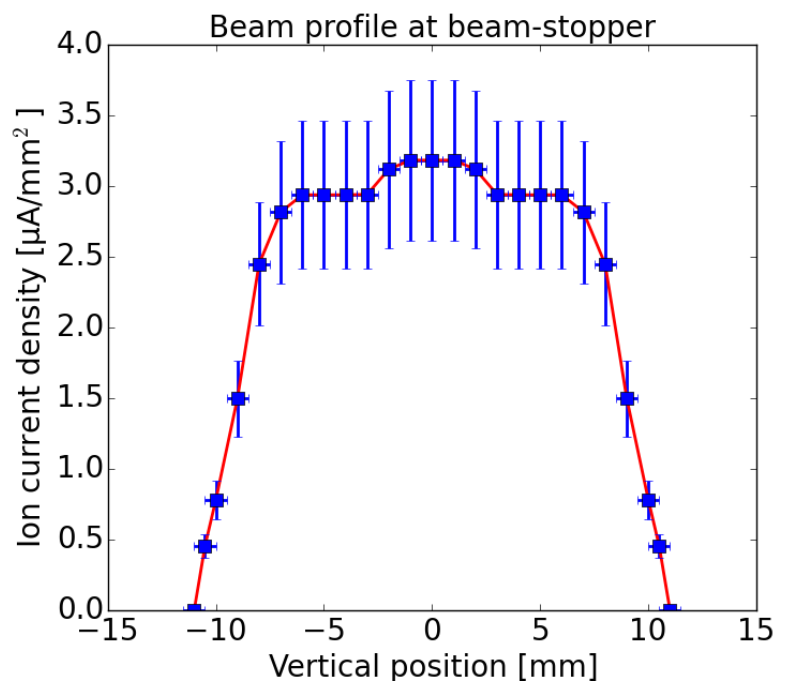

Figure 4: Beam profile at beam-stopper measured with Faraday cup. A line has been added to guide the eye. The ion current density is uniform near the beam axis within the uncertainty of the measurement, with an edge width of approx. $2 \mathrm{~mm}$. (Color online).

To measure the beam profile at the beam-stopper, a negatively biased aperture of $\varnothing 1 \mathrm{~mm}$ was installed onto the installed Faraday cup. The vertical position of the beam-stopper was then varied, to measure the offaxis current density of the beam, leading to line scan through the beam. Given the rotational symmetry of the ion source and extraction grids, the beam is also assumed to be cylindrically symmetric. The measured beam profile is shown in fig. 4 . The ion current density appears to have a uniform value around the beam axis of $\sim 3 \mu \mathrm{A} / \mathrm{mm}^{2}$. Beyond a diameter of $16 \mathrm{~mm}$, the ion current density drops quickly to zero. The width of the beam "edge" at this position is approximately $2 \mathrm{~mm}$.

The ion source behaves similarly when operating with hydrogen (protium), with the exception of the optimal 
operating pressure in the first differential pumping stage, which is increased to about $2 \times 10^{-4}$ mbar. The source cannot be ignited if helium is used as working gas. For this reason, in order to produce a He beam, the source was ignited with hydrogen and then helium was admixed, up to a flow ratio of $1: 1$. The He ions are then mass selected in the magnetic sector field which is explained in the following section (3.2). As with helium, the source does not ignite with argon. Similarly, the source must be ignited with hydrogen or deuterium and argon must then be admixed to produce an Ar beam. It must be noted that argon degrades the coating of the filament in a matter of hours. Because of this, long-term operation with argon requires intensive and continuous maintenance.

\subsection{Magnetic sector field deflection}

The ion beam deflection through the magnetic sector field in the dipole magnet was characterized with the current measurement at the target. The strength of the magnetic sector field was slowly increased while the current at the target was measured. When using deuterium, three discrete, strong peaks were observed, corresponding, respectively, to the three ion species $\mathrm{D}^{+}, \mathrm{D}_{2}{ }^{+}$and $\mathrm{D}_{3}{ }^{+}$. The same test was repeated with a hydrogen beam and a hydrogen-helium beam from a mixed plasma with a 7:3 H-to-He flow ratio. Again, three relatively large peaks were observed when using hydrogen, corresponding to $\mathrm{H}^{+}, \mathrm{H}_{2}{ }^{+}$and $\mathrm{H}_{3}{ }^{+}$, respectively. The peak corresponding to $\mathrm{He}^{+}$was also observed. If argon is used in the ion source, it is preferable to admix $\mathrm{D}$ rather than $\mathrm{H}$, since it is then easier to separate the resulting molecular ions $\left(\mathrm{ArD}^{+}\right.$ or $\mathrm{ArH}^{+}$, respectively) from the $\mathrm{Ar}^{+}$peak.

When the polarity of the dipole magnet's B-field was flipped and the current was measured on a Faraday cup mounted on the opposite exit (PERMEX exit in fig. 1), the sets of peaks observed were identical. This proves the symmetric behavior of the magnet.

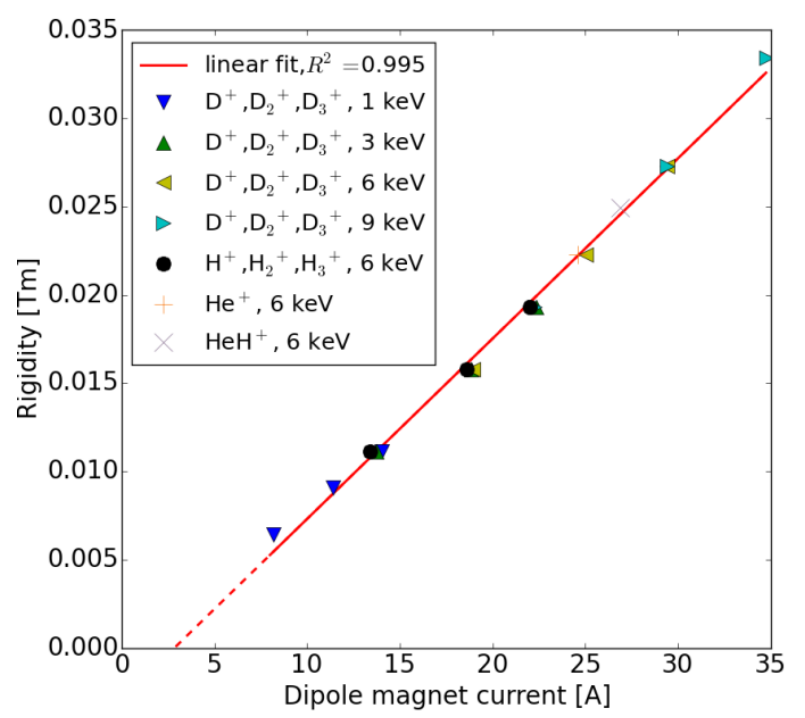

Figure 5: Rigidity of several ion species at energies ranging from 1 to $9 \mathrm{keV}$ plotted relative to the current applied to the dipole magnet at which the corresponding ion current peaks were measured. The rigidity should be directly proportional to the current through the magnet, which is proportional to the magnetic field. (Color online).

These tests were also repeated at differing ion energies. Figure 5 shows the rigidity of each ion, plotted as a function of the magnet current (which is proportional to the B-field) at which the corresponding peaks in the ion current measurement were observed. Hydrogen and helium ions with an energy of $6 \mathrm{keV}$ are shown, as are deuterium ions at energies of 1, 3, 6 and $9 \mathrm{keV}$. The rigidity of the ions scales linearly with the magnetic field at a given bending radius. In cases where the rigidity of the ions is approximately the same, for instance for $1 \mathrm{keV} \mathrm{D}_{3}{ }^{+}$ions, $3 \mathrm{keV} \mathrm{D}{ }^{+}$ions and $6 \mathrm{keV} \mathrm{H}^{+}$ions, it can be seen that the dipole magnet currents at which those peaks occur also coincide. It must be noted that the extrapolated linear regression from the data does not pass through the origin, as would be expected. This is believed to be due to the hysteresis of the dipole magnet: when magnetized from a zero-field value, the iron follows a non-linear magnetization curve. The magnetic field strength "lags behind" the current through the dipole magnet. 


\subsection{Beam emittance and ion optics}

In order to maximize the ion flux density arriving at the target, it was necessary to study and characterize the optical properties of the ion beam, as this would determine the final selection and placement of the ion lenses to be used.

As previously stated, the beam at the differential pumping stage and beyond is strongly space-charge compensated: the thermal electrons trapped in the potential well of the ion beam neutralize the beam, strongly reducing beam divergence due to Coulomb repulsion [6]. Electrostatic lenses are not used, because the unavoidable loss of space-charge compensation would increase divergence. Einzel lenses were tested at two positions in the ion optical system, marked in fig. 1, with positive and negative polarities of up to $6 \mathrm{kV}$, leading in all cases to a strong decrease in the ion current at the target due to loss of space-charge compensation.

The ion optical characteristics of the beam (beam profile and divergence) were studied at the exit of the dipole magnet. To visualize the beam, a Heraeus M382 Plus $^{2}$ Ce-doped quartz glass was installed at a distance of $16 \mathrm{~cm}$ from the dipole magnet's exit flange. When ions impinge on the glass, light is emitted due to fluorescence. In order to avoid charge accumulation on the surface of the glass, a fine nickel mesh was attached to the glass with Kapton ${ }^{3}$ tape. At the Mo aperture, the beam diverges equally in the horizontal and vertical plane and has a diameter of 16 $\mathrm{mm}$. As can be seen in fig. 6 , the focusing of the beam in the horizontal plane within the magnetic sector field leads to an elongated beam at the exit of the dipole magnet, with a narrow beam diameter of approximately $10 \mathrm{~mm}$. In the vertical plane, the beam is not focused and the beam diameter is $34 \mathrm{~mm}$. The real focusing effect at the position of the quartz glass in the horizontal plane is, therefore, from a diameter of $34 \mathrm{~mm}$ to one of $10 \mathrm{~mm}$. At positions further downstream from the dipole magnet, the beam waist

\footnotetext{
${ }^{2}$ Trademark of Heraeus Quarzglas GmbH \& Co. KG

${ }^{3}$ Trademark of E. I. du Pont de Nemours \& Co.
}

in the horizontal plane is reached and the beam again diverges in the horizontal plane. At the target, if no lenses are used, the beam divergence (and beam diameter) is larger in the horizontal plane than in the vertical plane. Asymmetric lenses such as quadrupole lenses, capable of applying different foci in the horizontal and vertical plane, must be used. Since the loss of space-charge compensation incurs in significant beam losses, magnetic lenses - which will not attract/repel the electrons trapped in the beam - are preferred to electrostatic lenses.

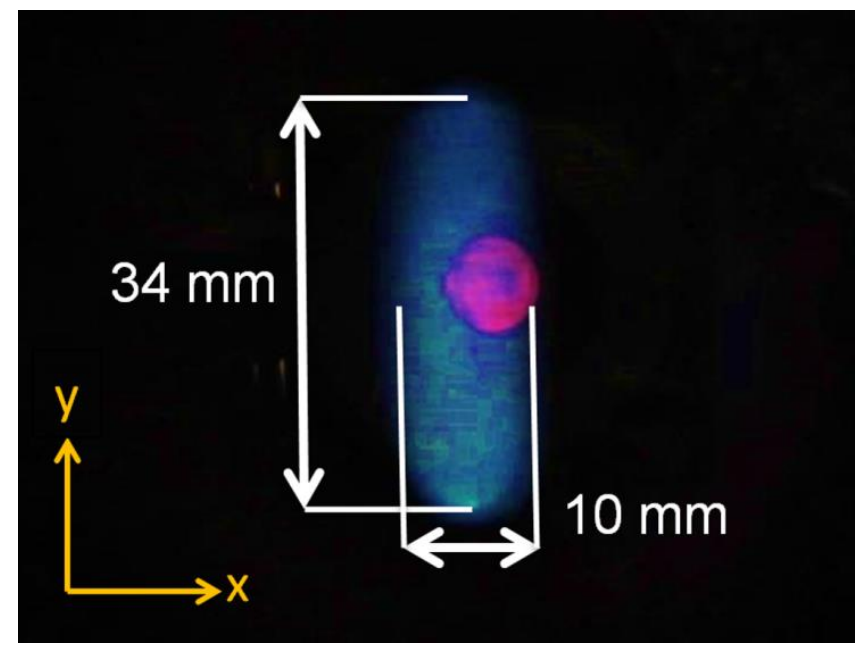

Figure 6: Beam profile of a $10 \mathrm{keV} \mathrm{D}_{3}{ }^{+}$beam on Ce-doped quartz glass $16 \mathrm{~cm}$ away from the exit of the dipole magnet. The beam is focused within the magnet in the deflection plane (x), leading to an astigmatic beam with a beam-waist smaller than $10 \mathrm{~mm}$ at the quartz glass. The red spot in the middle of the image is light from the ion source that is reflected inside the dipole magnet, and bears no relation to the beam spot. (Color online).

The measurement of the beam emittance was necessary in order to design and commission the quadrupole lenses. The emittance ellipse was measured by the pepper-pot method, as described in $[9,10]$. A plate with equally spaced holes (pepper-pot) was placed at the exit flange of the dipole magnet. As the beam passed through the holes, the beamlets produced individual beam footprints on the quartz glass. With this information, the angular distribution of the beam at the position of each hole on the pepper pot was measured, from which the beam ellipse is reconstructed to obtain the Twiss parameters [11]. Figure 7 shows the reconstructed ellipse in the 
horizontal and vertical planes along with the values of the Twiss parameters at the exit flange of the dipole magnet.
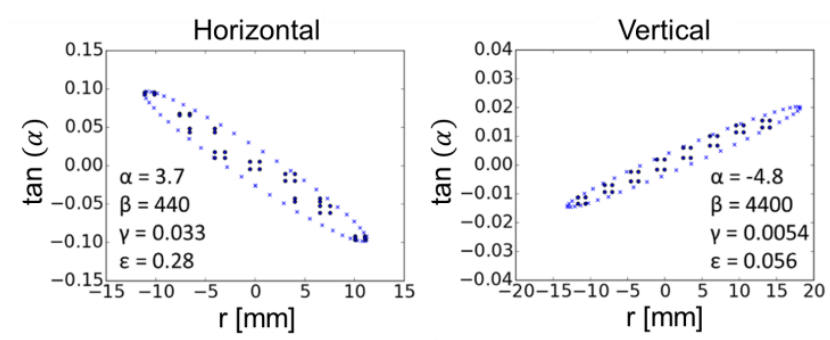

Figure 7: Reconstructed beam ellipses from a $10 \mathrm{keV} \mathrm{D}_{3}{ }^{+}$beam in the horizontal (left) and vertical (right) planes at the exit flange of the dipole magnet with the calculated Twiss parameters. Dark blue dots correspond to the measured points. Light blue crosses indicate the circumscribing ellipse. The beam is converging in the horizontal plane (negative angles for positive distances), while diverging in the vertical plane (positive angles at positive distances). The area of the ellipse is $\varepsilon \times \pi$ in mm $\times$ rad. (Color online).

The measured Twiss parameters were used in ion optical numerical calculations based on the transfer matrix method to design the magnetic quadrupole doublet lens. A custom-written code optimized the magnetic fields of the quadrupole doublet in order to maximize the ion flux at the target. A single quadrupole focuses the beam in one plane while simultaneously de-focusing in the perpendicular plane. The horizontal and vertical focal points are, therefore, not independent. A combination of two allows for an additional degree of freedom, so that both focal points may be adjusted freely, and so net focusing in both planes can be achieved. Calculations showed the use of such a magnetic quadrupole could entail an increase of ion current at the target by up to a factor of 20 compared to values without installing the lens. Fig. 8 shows calculated trajectories of the edge of the beam in the horizontal and vertical plane between the dipole magnet and the target if a fully-optimized quadrupole doublet lens is used. Based on these results, a magnetic quadrupole doublet model 'QM-60.100' from D-Pace ${ }^{4}$ with a bore diameter of $60 \mathrm{~mm}$ and a peak pole-tip field of $0.15 \mathrm{~T}$ was ordered and installed. The quadrupole doublet is situated outside of the

\footnotetext{
${ }^{4}$ Registered trademark
}

vacuum system. The position of the quadrupole lens system is shown in fig. 1 . As is visible in fig. 8, the beam trajectories may expand significantly within the quadrupole lens during focusing. In order to prevent contact with the walls of the vacuum tube connecting the dipole magnet and the target chamber under a wide range of possible operating conditions, the $321 \mathrm{~mm}$ long vacuum tube used to connect the two systems was custom built to have a larger internal diameter $(\varnothing$ $49.8 \mathrm{~mm}$ instead of the nominal $\emptyset 38 \mathrm{~mm}$ for a CF40 tube). In agreement with the simulations, the highest current at the target chamber was measured when the quadrupole closest to the dipole magnet was configured to vertically focus the beam and the quadrupole closest to the target chamber focused the beam horizontally. The position of the quadrupoles along the beam axis was varied by $\pm 40 \mathrm{~mm}$ from their nominal position. Experimentally, this was not seen to have any significant effect on the maximum ion current measured at the target chamber.

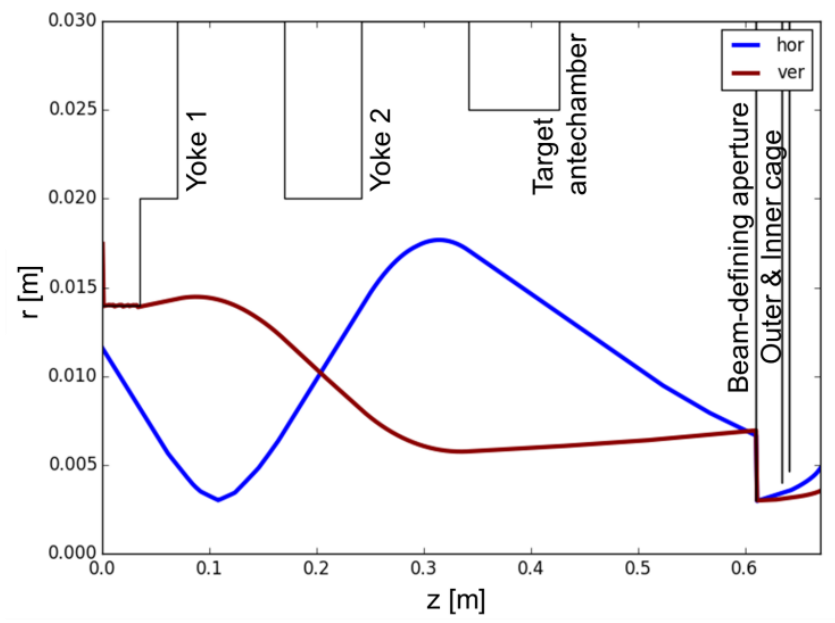

Figure 8: Numerical calculations of the edge of the beam trajectory through a magnetic quadrupole doublet between the dipole magnet $(\mathrm{z}=0 \mathrm{~m})$ and the target $(\mathrm{z}=0.6 \mathrm{~m})$ in the horizontal (hor) and vertical planes (ver) when focusing a $6 \mathrm{keV} \mathrm{D}_{3}{ }^{+}$beam. Between the grounded inner cage and the target (right edge of the image), the ions are decelerated to an impinging ion energy of $600 \mathrm{eV} / \mathrm{D}$. The first quadrupole along the $\mathrm{z}$ axis focuses vertically with a pole tip field of $0.041 \mathrm{~T}$ and the second focuses horizontally with a pole tip field of $0.073 \mathrm{~T}$. (Color online).

When focusing a $6 \mathrm{kV} \mathrm{D}_{3}{ }^{+}$ion beam with the quadrupole doublet lens, an increase of a factor of 4 in the ion current was achieved. The discrepancy 
between this and the factor of 20 increase predicted by the calculations is attributed to two factors. Firstly, the ion density of the beam was assumed to be uniform in the simulations and under this assumption, when no quadrupole lens is used, a large part of the ion current would impinge on the stainless steel walls of the vacuum chambers and the beam-defining aperture. If the ion density is not uniform, but instead higher near the center of the beam, then a larger fraction of the beam would reach the target without the use of a lens, in which case the relative increase in ion current at the target due to the addition of said lens would be smaller than predicted. Secondly, the focusing effect of the quadrupole lens is highly sensitive to the correct alignment of each of the two quadrupoles. It was observed that a sub-mm precision is required to adequately focus the beam. This was not foreseen when designing the support structure for the quadrupole doublet lens, and it is possible that the lens is not yet sufficiently well aligned with the target to achieve the optimal performance. If this is the case, then better alignment may provide larger ion currents at the target. In view of this, a fine-adjustment support structure is to be installed in the future.

\subsection{Beam footprint and neutral population at the target}

The beam footprint at the target was measured without focusing from the quadrupole lens by exposing samples consisting of a 70-nm-thick layer of amorphous hydrogenated carbon $(\mathrm{a}-\mathrm{C}: \mathrm{H})$ on a $\mathrm{Si}$ substrate to a deuterium ion beam. For these samples, a change in layer thickness of a few nanometers leads to a change in color. This change in layer thickness was quantitatively measured applying ellipsometry [12]. Two a-C:H samples were eroded by a $6 \mathrm{keV} \mathrm{D}_{3}{ }^{+}$ beam when using a $\varnothing 6 \mathrm{~mm}$ beam-defining aperture. The quadrupole doublet lens was not employed. Figure 9 shows the 2D-ellipsometry measurements and horizontal line scans of the samples, from which $20 \mathrm{~nm}$ on average were eroded in each case. On both samples, the ion beam footprint corresponds to the horizontally-elongated ellipse. Since the beam diverges differently in the horizontal and vertical planes between the beam-defining aperture and the target (stronger divergence at the target in the horizontal plane), the footprint of the beam is elliptical and the surface area of the beam-defining aperture cannot be taken as the surface area of the beam footprint. A second vertically elongated ellipse is also visible on the sample. This ellipse is assumed to be caused by a small percentage of neutral particles which impinge on the a-C:H layer, altering the optical properties of the surface. The neutral population of the ion beam is quantified at the end of this section.
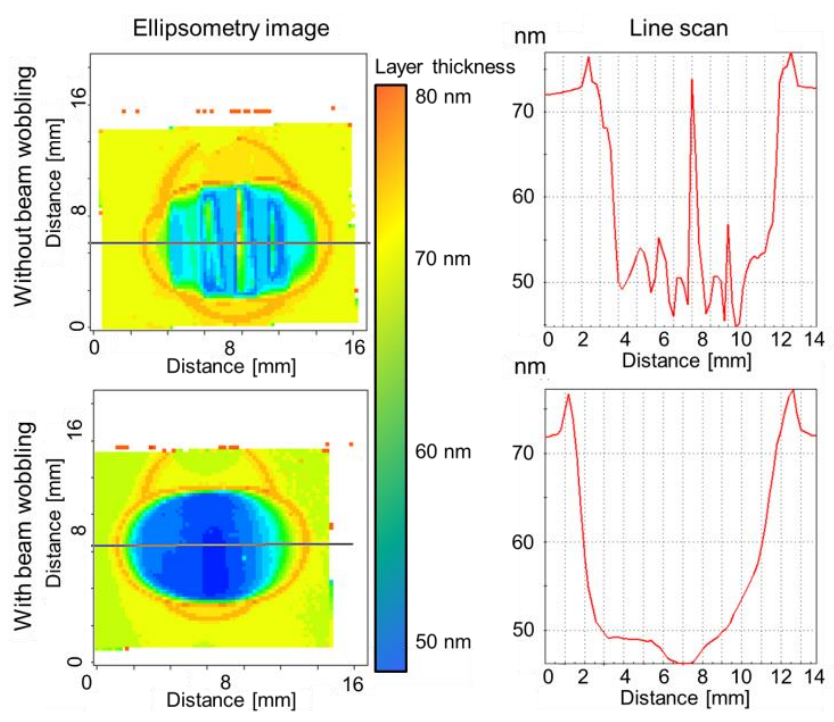

Figure 9: Ellipsometry measurements of the beam footprint at the target obtained by exposing a 70-nm-thick a-C:H layer on Si to a 6 $\mathrm{keV} \mathrm{D}_{3}{ }^{+}$beam, when using a $\varnothing 6 \mathrm{~mm}$ beam-defining aperture. The images on the left show a 2D pattern of the a-C:H layer erosion. The images on the right are line scans along the line indicated in the left figure. In the lower pair of images, the ion beam was scanned over the beam- defining aperture. For details please refer to the text. (Color online).

In the topmost image, evenly-spaced vertical lines are visible along the horizontal axis of the sample. This is due to areas with different erosion. These vertical lines, of approximately $1 \mathrm{~mm}$ in width, are a consequence of the horizontal beam focusing effect of the dipole magnet: after passing through the magnetic sector field the beam is focused in the deflection (horizontal) plane, forming an image of the openings of the last (grounded) of the three grids (described in section 2) located at the exit of the ion source. No image is formed in the vertical plane, because in this 
plane the focal point is not located upstream from the target chamber. Since the holes of the grids are vertically aligned, they appear as vertical stripes on the sample. Due to the construction of the grids, it is not possible to rotate them in a way that will eliminate the stripes. To accomplish this, new grids with an altered geometry would have to be manufactured. One way to avoid the formation of these vertical stripes is to periodically vary the current through the dipole magnet back and forth by a small amount, typically \pm $0.2 \mathrm{~A}$, thereby "sweeping" the ion beam over the beam-defining aperture. This was performed when eroding the second a-C:H sample (lower images of figure 9). The increase in layer thickness at the edge of the beam-spot visible in both images (orange) is an artifact of the ellipsometry measurement. D implantation into the a-C:H layer leads to a change of the optical properties of the layer, which are interpreted by the analysis software as an increase of the layer thickness [12].

When using the quadrupole doublet lens to focus the beam, inhomogeneities in the beam footprint at the target are also observed, although depending on the lens focusing effect, these may not necessarily take the shape of vertically elongated lines. As was the case without focusing with the quadrupole lens, oscillating the current through the dipole magnet to sweep the beam over the beam-defining aperture also helps to "smooth out" the inhomogeneities in the beamfootprint at the target. Another alternative to reduce said inhomogeneities would be to induce vertical and horizontal beam-sweeping by operating each of the four coils of the quadrupoles separately. However, this method would compromise the beam focusing effect of the quadrupole lens which is responsible for the increase in ion flux to the target. Since this was the main application of the quadrupole lens, this method to homogenize the beam footprint was not attempted. Electrostatic beam-sweeping with capacitor plates was also not implemented, as this would have led to a loss of space-charge compensation in the ion beam, as was evidenced in the tests with einzel-lenses in section 3.3.
Samples coated with a-C:H layers were also used to measure the surface area of the beam footprint at the target, which is required together with the impinging ion current to calculate the ion flux density (and fluence) at the target.

Table 1 shows the measured surface areas eroded by a $6 \mathrm{keV} \mathrm{D}{ }^{+}$beam under various angles of incidence. To prevent part of the beam from extending beyond the target and impinging on the inside of the double cage, the appropriate beam-defining aperture had to be chosen for each angle of incidence at which a measurement took place.

\begin{tabular}{l|l|l} 
Angle $\left[^{\circ}\right]$ & Surface area $\left[\mathrm{cm}^{2}\right]$ & Aperture $[\mathrm{mm}]$ \\
\hline 0 & 0.51 & $\emptyset 6$ \\
45 & 0.75 & $3 \times 6$ \\
60 & 0.82 & $2 \times 6$ \\
75 & 0.94 & $1.5 \times 6$
\end{tabular}

Table 1: Measured surface area of beam footprint on a-C:H samples eroded under varying angles of incidence by a $6 \mathrm{keV} \mathrm{D}_{3}{ }^{+}$ beam. The required aperture was chosen for each angle to ensure that the beam only impinged on the sample.

Ions can be neutralized in-flight in between the ion source and the target by charge exchange collisions. Neutral particles produced between ion source and dipole magnet will not be deflected and, therefore, will not reach the target. If experiments with low-energy ions are desired, the target will be biased to decelerate the ions. However, high energy neutral particles produced between the exit of the dipole magnet and the target double cage will not be decelerated and may impinge on the target with the full primary energy. Given the difference in impinging energy, the effect of these impinging neutral particles on the sample can, in some cases, be of comparable magnitude to the effect of the much higher flux of low-energy ions. Therefore, if the impinging neutral particles are not taken into account, this neglect can severely compromise the interpretation of the experimental results. In order to assess the impact of the neutral particles, the neutral population of the beam at the target was quantified. As a first step, the neutral population was estimated analytically by equating the neutral population with the probability of neutralization $P$, which is taken as a first approximation as: 


$$
P=\sigma n L,
$$

where $\sigma$ is the charge exchange cross section. No charge exchange cross section data could be found for $\left[\mathrm{D}_{3}{ }^{+}, \mathrm{D}_{2}\right]$, but as a first approximation the cross section is assumed to be similar to that of $\left[\mathrm{D}^{+}, \mathrm{D}_{2}\right]$, which for $3 \mathrm{keV}\left[\mathrm{D}^{+}, \mathrm{D}_{2}\right]$ is $2 \times 10^{-19} \mathrm{~m}^{2}$, taken for $\mathrm{H}$ from [13] with the corresponding energy correction. The density of neutral gas between dipole magnet and target, $n$, is derived from the gas pressure by applying the ideal gas law at room temperature. As a conservative assumption, the neutral gas density was calculated with the pressure in the dipole magnet $\left(5 \times 10^{-6} \mathrm{mbar}\right)$, which is higher than the pressure in the target chamber $\left(5 \times 10^{-7}\right.$ mbar), leading to $\mathrm{n}=1.2 \times 10^{17} \mathrm{D}_{2} / \mathrm{m}^{3} . L$ is the distance between dipole magnet and target, which in the case of SIESTA is $0.6 \mathrm{~m}$. By applying equation 1, the neutral population is estimated as $1.4 \%$.

To measure the neutral fraction in the beam a thin gold layer on a silicon substrate was exposed to a D ion beam. Physical Vapor deposition was used to produce a sample consisting of a $45 \mathrm{~nm}$ Au layer on a smooth Si substrate, with a $5 \mathrm{~nm}$ Ti interlayer included to ensure adequate adhesion of the Au layer and to act as a diffusion barrier between $\mathrm{Au}$ and $\mathrm{Si}$. The 2dimensional thickness distribution of the Au layer was measured by Rutherford Backscattering Spectrometry (RBS) with $2 \mathrm{MeV} \mathrm{He}^{+}$ions with an analysis beamspot size of approximately $1 \times 1 \mathrm{~mm}^{2}$. The maximum variation of the thus-determined Au-layer thickness across the surface of the sample was $0.5 \mathrm{~nm}$, indicating the very good homogeneity of the Au layer. The sample was subsequently exposed at SIESTA to a neutral deuterium beam of $1.2 \mathrm{keV} / \mathrm{D}$ by employing an extraction potential of $3.6 \mathrm{kV}$ to extract $\mathrm{D}_{3}{ }^{+}$ions and positively biasing the target to $3.8 \mathrm{kV}$. By biasing the target in this fashion, all deuterium ions are deflected away from the target and do not reach the sample, while the fraction of neutral particles in the beam is not affected by target biasing and thus impinges with full extraction energy on the sample. The sample was exposed in this manner for 32 hours. During this exposure, the $\mathrm{D}_{3}^{+}$ion current was measured periodically by rotating the target platform $180^{\circ}$ and allowing the beam to impinge on the backside of the grounded heating cup, which is connected to the current measurement for this purpose. The current was determined to be constant, with a variation below $1 \%$ during exposures.

After erosion, the Au-layer thickness was again measured by RBS and the eroded Au-layer thickness was determined. Measurements were carried out in 1 $\mathrm{mm}$ steps along the longitudinal midplane of the sample and perpendicular to the midplane at the position of the measured erosion maximum. The measured beam spots are marked with an $\mathrm{X}$ in fig. 10 . The sputter yield for $1.2 \mathrm{keV} / \mathrm{D}$ on $\mathrm{Au}$ is 0.0305 and was taken from [3]. With this method, the neutral fluence at each of the positions measured with RBS was calculated. These data were contrasted with the exposure time and average ion current to determine the neutral population of the ion beam at said positions. The neutral population is calculated as the number of energetic neutral $\mathrm{D}$ atoms divided by the number of $\mathrm{D}$ atoms arriving in form of ions, i.e., in the case of $\mathrm{D}_{3}{ }^{+}$ions the latter number is three times the ion flux. The erosion - and thus neutral population - at other positions on the sample was interpolated from these measurements by applying the tensor-product method. The maximal measured thickness change was $41.7 \mathrm{~nm}$ corresponding to $93 \%$ of the 45 -nm-thick $\mathrm{Au}$ layer. At its maximum, the neutral population is of $1.5 \%$, while the average among the measurement points is of $0.8 \%$. As shown in figure 10 , the neutral beam is predominantly concentrated off-center on the upper-right quadrant of the sample. The beam footprint of the neutral "beam" differs from that of the ion beam under equivalent exposure conditions (i.e., if the target were not biased) because the ion beam would be focused by the quadrupole doublet lens, whereas in the case of the neutral "beam", those ions neutralized between the exit of the dipole magnet and the first quadrupole (the majority, since the pressure in the dipole magnet is larger than in the target chamber) would not be focused at all. Those neutralized between the quadrupoles would be focused by the first one and not by the second one, and only those ions 
neutralized between the second quadrupole and the target would be focused in the same way as the ion beam. The neutral "beam" can be considered a combination of these three beams. Due to small errors in the adjustment of the quadrupoles (mentioned briefly at the end of section 3.3), the axes of the two quadrupoles and the exit of the dipole magnet do not completely coincide, so the overall neutral "beam" trajectory is slightly off-axis with regard to the ion beam. It is worth noting that this is not the case when the quadrupole lenses are not employed, as evidenced in fig. 9, where the neutral beam footprint coincides with that of the ions.

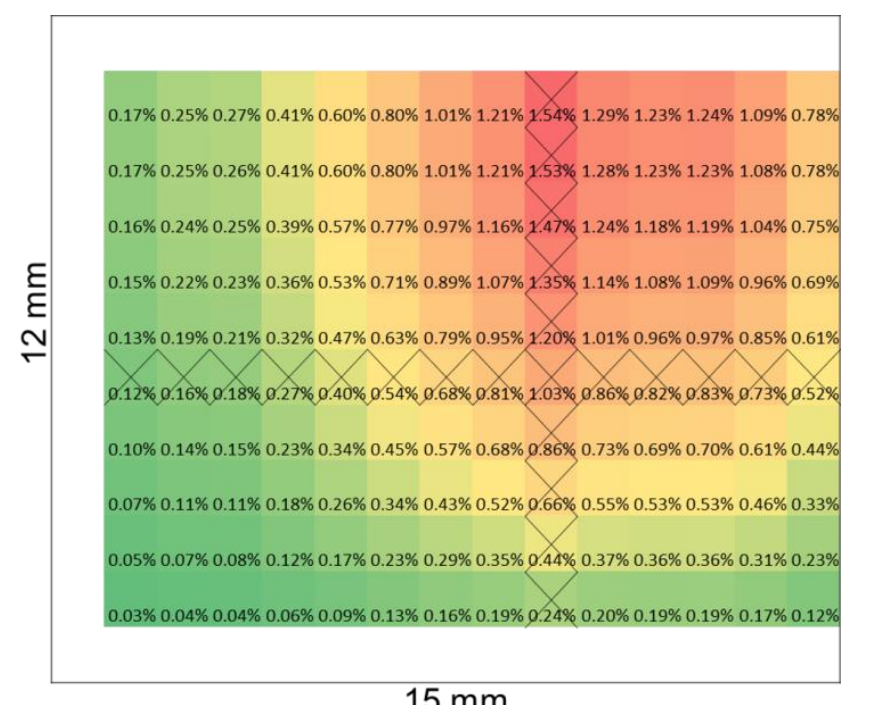

$15 \mathrm{~mm}$

Figure 10: Position-resolved neutral population at the target measured on a $12 \times 15 \mathrm{~mm}$ sample. The percentage in each cell represents the neutral population of the impinging beam on that $1 \times 1 \mathrm{~mm}$ cell. Red corresponds to areas where the neutral population is larger than $1 \%$. Yellow indicates it is between $0.5 \%$ and $1 \%$. Green indicates the neutral population is below $0.5 \%$. X marks the positions where the neutral population was measured by RBS. The neutral populations at all other positions were interpolated by the tensor-product method from the measured data. (Color online).

The neutral population of the ion beam has been estimated to be approximately $1 \%$ under the given exposure conditions. Based on this information, e.g., in the case of sputtering at low incident ion energies, if the sputter yield of the high-energy neutrals is larger than the sputter yield of the low-energy ions by a factor of 100 or more, the neutral particles will substantially contribute to or even dominate the sputtering process, and therefore cannot be neglected. In such cases, it may be necessary to perform a dedicated measurement of the neutral population analogous to this one under the required experimental conditions.

\section{Erosion of Au bulk samples}

\subsection{Experimental procedure}

Gold was chosen as target material for the characterization of SIESTA due to three material properties: firstly, due to the high molar mass of $\mathrm{Au}$, significant weight loss can be achieved with moderate erosion. Secondly, the sputter yield (SY) of D on Au in the $\mathrm{keV}$ range is relatively high compared with that of its neighbors in the periodic table, so a comparatively lower fluence is required to achieve the desired erosion (e.g., SY of $2 \mathrm{keV} \mathrm{D}$ on $\mathrm{Au}$ is 0.037 ; $\mathrm{SY}$ of $2 \mathrm{keV} \mathrm{D}$ on W is 0.005 [2]). Thirdly, since Au is a noble metal, ex-situ weight loss measurements can be directly performed without the need to consider the possible formation of an oxide layer.

Four pure gold bulk samples of $12 \times 15 \mathrm{~mm}$ were mechanically polished to a mirror finish and were eroded by a $6 \mathrm{keV} \mathrm{D}_{3}{ }^{+}$ion beam (yielding $2 \mathrm{keV} / \mathrm{D}$ ) at room temperature under normal incidence. All samples were weighed before and after exposure in an ex-situ microbalance. The sputter yield was determined by mass loss, and was compared to data from literature and simulations with the SDTrimSP code [14]. Said simulations were performed with the standard parameters (Gauss-Mehler quadrature integration method with 8 pivots and ipot set to 1 ).

To include the slight day-to-day variability in the operation of the ion source (slightly different pressure or gas flow in the source, source filament aging...), the samples were exposed on different days. On each day, the samples were weighed ex-situ in the morning, exposed during the day and weighed again after exposure in the evening. A reference sample was weighed alongside the exposed sample on each 
occasion to correct for potential drift of the ex-situ microbalance measurement during the day due to environmental factors such as temperature or humidity. The samples were weighed several times on each occasion. The balance was also tared between measurements.

\subsection{Sputter yield results}

The sputter yield was calculated by applying:

$$
S Y=\frac{\Delta \text { mass }[\mathrm{g}] \times N_{A}[\text { atoms } / \mathrm{mol}]}{N_{\text {ions }} \times M(A u)[\mathrm{g} / \mathrm{mol}]} .
$$

Here $\Delta$ mass is the mass loss due to erosion, $N_{A}$ is Avogadro's number, $M(A u)$ is the standard atomic weight of gold and $N_{\text {ions }}=3 \times Q[\mathrm{C}] / e[\mathrm{C}]$ is the total amount of impinging particles. $Q$ is the collected charge at the target and $e$ is the elementary charge.

The results are shown in figure 11 . The average of the sputter yields obtained experimentally in this work is approximately $15 \%$ higher than the yield calculated by SDTrimSP, and approximately $25 \%$ higher than the average of the data points from literature [2, 15], though it must be noted that these show a significant scatter. The value given by SDTrimSP is within the scatter of Behrisch's data points [2] and shows good agreement with Bay [15]. It was decided to take the SDTrimSP value as the benchmark for SIESTA. Given that the experimental data points from SIESTA show comparatively low scatter $(<4 \%)$, it is unlikely that the deviation of $15 \%$ between the experimentallyobtained average and the SDTrimSP sputter yield is due to an issue with reproducibility at SIESTA. The deviation could possibly be attributed to a systematic error in the current measurement. Sputtering due to the presence of energetic neutral particles (explained and quantified in section 3.4) would also contribute to this error in measurement (though it would not be sufficient on its own to explain the discrepancy), as could a possible systematic error in the weight loss measurement.

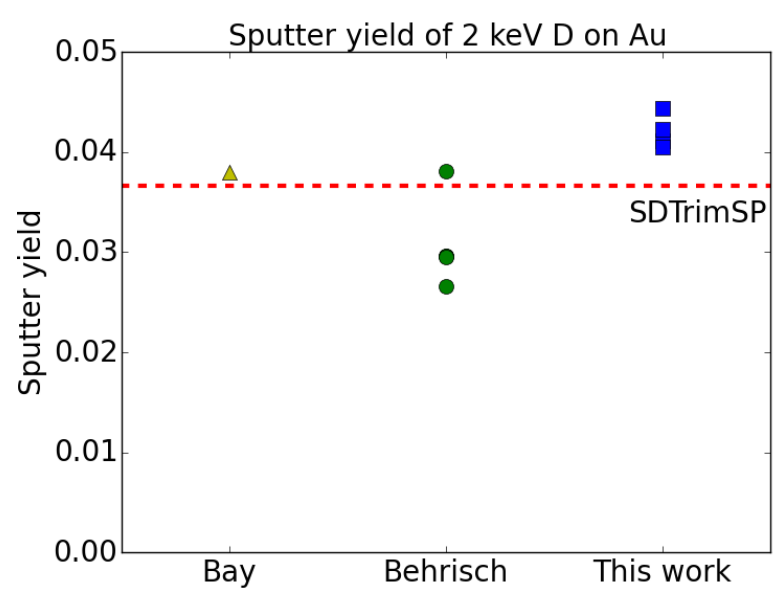

Figure 11: Sputter yield of bulk Au samples exposed to a $2 \mathrm{keV} \mathrm{D}$ beam $\left(6 \mathrm{keV} \mathrm{D}_{3}{ }^{+}\right)$at SIESTA, determined by ex-situ weight loss. An SDTrimSP simulation (red dashed line) and literature values from Behrisch [2] and Bay [15] are included for comparison. The uncertainty of the experimental sputter yield measurements ("This work") is contained within the size of the symbols. (Color online).

\section{Summary}

SIESTA (Second Ion Experiment for Sputtering and TDS Analysis) is a fully operational setup with a highcurrent ion source which is suited for well-defined ioninduced erosion and retention studies. It can supply a mass-filtered ion beam of $\mathrm{H}, \mathrm{D}, \mathrm{He}$ or Ar ions with energies of $200 \mathrm{eV}-10 \mathrm{keV}$ with ion flux densities (in the case of $6 \mathrm{keV} \mathrm{D}_{3}^{+}=2 \mathrm{keV} / \mathrm{D}$ ) of up to several $10^{19}$ atoms $\mathrm{m}^{-2} \mathrm{~s}^{-1}$ at the target when employing the quadrupole lens to focus the beam, as listed in table 2 for several ion species and energies. Mass filtering is achieved with a dipole magnet capable of deflecting a $10 \mathrm{keV} \mathrm{Ar}^{+}$beam. The typical beam footprint measures $0.5 \mathrm{~cm}^{2}$ in the case of a $6 \mathrm{keV} \mathrm{D}_{3}{ }^{+}$beam at $0^{\circ}$ incidence to the target (i.e., normal to the sample surface) with a $\varnothing 6 \mathrm{~mm}$ beam-defining aperture. The sample may be rotated to study angle-dependent effects and sample temperatures of up to $1300 \mathrm{~K}$ are achieved via electron impact heating on the sample backside. The temperature is measured with an infrared pyrometer and a type-K thermocouple. Samples may be inserted into the target chamber with the use of a load-lock. Thermal desorption spectrometry is available in a dedicated vacuum chamber located below the load-lock for in-vacuo 
retention studies, though this capability of SIESTA has not yet been tested.

\begin{tabular}{l|l|ll} 
Ion species & Energy at target [eV/atom] & Flux density $\left[10^{19}\right.$ atoms $\left.\mathrm{m}^{-2} \mathrm{~s}^{-1}\right]$ & $\begin{array}{c}\text { With } \\
{\left[10^{19} \text { atoms } \mathrm{m}^{-2} \mathrm{~s}^{-1}\right]}\end{array}$ \\
\hline $\mathrm{H}^{+}$ & 6000 & 0.22 & \\
$\mathrm{H}_{2}^{+}$ & 3000 & 0.37 & \\
$\mathrm{H}_{3}^{+}$ & 2000 & 0.94 & \\
$\mathrm{D}^{+}$ & 6000 & 0.28 & \\
$\mathrm{D}_{2}^{+}$ & 3000 & 0.41 & 6.0 \\
$\mathrm{D}_{3}^{+}$ & 2000 & 1.5 & \\
$\mathrm{D}_{3}^{+}$ & 1000 & 1.3 & 3.7 \\
$\mathrm{D}_{3}^{+}$ & 200 & 1.0 & \\
$\mathrm{He}^{+}$ & 6000 & 0.45 & 0.37 \\
$\mathrm{Ar}^{+}$ & 5000 & 0.10 &
\end{tabular}

Table 2: Performance data for SIESTA. Ion flux densities at the target are listed for varying ion species and energies at normal incidence. In the case of $6 \mathrm{keV} \mathrm{D}_{3}{ }^{+}$(equaling $\left.2 \mathrm{keV} / \mathrm{D}\right), 600 \mathrm{eV} \mathrm{D}_{3}{ }^{+}(200 \mathrm{eV} / \mathrm{D})$ and $5 \mathrm{keV} \mathrm{Ar}$, flux densities achieved when using the quadrupole doublet lens to focus the beam at the target are also listed.

In this work, the device was assembled and extensively characterized. Effective mass-filtering was tested for a wide range of ion species and energies. The beam emittance was measured at the exit of the dipole magnet. Based on these measurements, the ion beam trajectories were calculated and a quadrupole doublet lens was designed and commissioned. The lens was installed between the dipole magnet and target chamber and is used for simultaneous focusing of the ion beam in the horizontal and vertical plane. If no quadrupole lens is used, the flux density at the target at SIESTA is comparable to that of its predecessor, the HSQ [4]. If the quadrupole lens is employed, the flux density at the target is increased by up to a factor of 4 compared to the HSQ. Table 2 lists deuterium and argon ion flux densities with and without the focusing effect of the quadrupole lens.

The beam footprint at the target was measured with ellipsometry after erosion of 70-nm-thick amorphous hydrogenated carbon films on $\mathrm{Si}$ substrates. A homogeneous beam footprint at the target was achieved by sweeping the ion beam over the beamdefining aperture by oscillating the current passing through the dipole magnet. Sample heating was tested on W samples up to $1300 \mathrm{~K}$ and a push-rod mechanism was introduced to improve the thermocouple temperature measurement. The in-situ magnetic suspension balance - while installed - is not yet ready for general use. In the near future, thermal and electromagnetic shielding may have to be installed to minimize the influence of external perturbations on the in-situ weight loss measurement.

The spatially-resolved neutral population within the beam at the position of the target was measured by eroding a 50-nm-thick Au film on a Si substrate and measuring the change of the layer thickness by Rutherford Backscattering Spectrometry (RBS). These experiments resulted in a maximum neutral population of $1.5 \%$ and an average population over the measured locations of $0.8 \%$. Ex-situ weight loss measurements

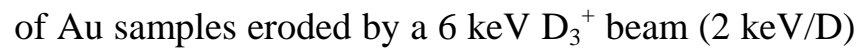
were performed. The sputter yield of pure $\mathrm{Au}$ under these exposure conditions was determined from the ex-situ weight loss measurements. The results agree within $15 \%$ with the sputter yield calculated by the sputtering code SDTrimSP [14].

\section{Supplementary material}

The supplementary material of this article includes more detailed descriptions of the technical setup and of the characterization of the ion source, beam optics, ion current measurement and in-situ magnetic suspension balance.

\section{Acknowledgements}

The authors would like to express their gratitude to T. da Silva, A. Manhard, U. von Toussaint, H. Greuner, 
T. Höschen, J. Roth, L. Seidl and M. Reisner for their helpful feedback as well as to A. Friedrich, A. Weghorn, M. Fußeder, J. Dorner and the E2M workshop for their continued support.

This work has been carried out within the framework of the EUROfusion Consortium and has received funding from the Euratom research and training programme 2014-2018 under grant agreement No 633053. The views and opinions expressed herein do not necessarily reflect those of the European Commission.

\section{References}

[1] J. Roth, E. Tsitrone, A. Loarte, Th. Loarer, G. Counsell, R. Neu, V. Philipps, S. Brezinsek, M. Lehnen, P. Coad, Ch. Grisolia, K. Schmid, K. Krieger, A. Kallenbach, B. Lipschultz, R. Doerner, R. Causey, V. Alimov, W. Shu, O. Ogorodnikova, A. Kirschner, G. Federici, A. Kukushkin, and EFDA PWI Task Force, ITER PWI Team, Fusion for Energy, ITPA SOL/DIV. Recent analysis of key plasma wall interactions issues for ITER. Journal of Nuclear Materials, 390-391:1-9, 2009.

[2] R. Behrisch and W. Eckstein. Sputtering by Particle Bombardment IV, volume 110 of Topics in Applied Physics. Springer Verlag, Berlin, 2007.

[3] W. Eckstein. Sputtering yields. In R. Behrisch and W. Eckstein, editors, Sputtering by Particle Bombardment IV, volume 110 of Topics in Applied Physics, chapter Sputtering Yields, pages 33-187. Springer Verlag, Berlin, 2007.

[4] W. Eckstein, C. García-Rosales, J. Roth, and W. Ottenberger. Sputtering data. IPP Report 9/82, MaxPlanck-Institut für Plasmaphysik (Ed.) http: //hdl.handle.net/11858/00-001M-0000-0027-6324-6, 1993.

[5] R. C. Davis, O. B. Morgan, L. D. Stewart, and W. L. Stirling. A multiampere DuoPIGatron ion source. Review of Scientific Instruments, 43(2):278-283, 1972.

[6] N. Chauvin, O. Delferrière, R. Duperrier, R. Gobin, P. A. P. Nghiem, and D. Uriot. Transport of intense ion beams and space charge compensation issues in low energy beam lines (invited). Rev. Sci. Instrum., 83(2):02B320, 2012.
[7] C. D. Child. Discharge from hot $\mathrm{CaO}$. Physical Review (Series I), 32(5):492-511, 1911.

[8] E Speth. Neutral beam heating of fusion plasmas. Reports on Progress in Physics, 52(1):57, 1989.

[9] J.G. Wang, E. Boggasch, P. Haldemann, D. Kehne, M. Reiser, T. Shea, and D.X. Wang. Performance characteristics of a variable-perveance gridded electron gun. In IEEE Transaction on Electron Devices, volume 37, pages 2622-2628. IEEE, 1990.

[10] J.G. Wang, D.X. Wang, and M. Reiser. Beam emittance measurement by pepper-pot method. Nucl. Instrum. Methods Phys. Res., Sect. A 307:190-194, 1991.

[11] M.G. Minty and F. Zimmermann. Measurement and Control of Charged Particle Beams. Springer Berlin Heidelberg, 2003.

[12] T. Schwarz-Selinger, A. von Keudell, and W. Jacob. Novel method for absolute quantification of the flux and angular distribution of a radical source for atomic hydrogen. Journal of Vacuum Science \& Technology A: Vacuum, Surfaces, and Films, 18(3):995-1001, 2000.

[13] R. Janev, W. Langer, K. Evans, and D. Post. Elementary Processes in Hydrogen-Helium Plasmas, volume 4. Springer-Verlag Berlin Heidelberg, 1 edition, 1987.

[14] A. Mutzke, R. Schneider, W. Eckstein, and R. Dohmen. SDTrimSP Version 5.00. IPP Report 12/08, Max-Planck-Institut für Plasmaphysik (Ed.) http://hdl.handle.net/11858/00-001M-0000-0026EAF9-A, 2011.

[15] H. L. Bay, J. Roth, and J. Bohdansky. Light-ion sputtering yields for molybdenum and gold at low energies. Journal of Applied Physics 48, 4722, 1977. 